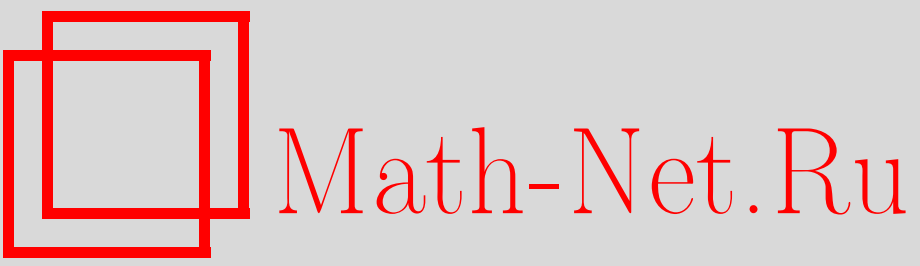

И. К. Костов, В. Б. Петкова, Объемные корреляционные функции в двумерной квантовой гравитации, ТМФ, 2006, том 146, номер 1, 132-145

DOI: https://doi.org/10.4213/tmf2014

Использование Общероссийского математического портала Math-Net.Ru подразумевает, что вы прочитали и согласны с пользовательским соглашением

http://www.mathnet.ru/rus/agreement

Параметры загрузки:

IP : 54.224 .135 .184

26 апреля 2023 г., 18:00:28 
ТЕОРЕТИЧЕСКАЯ

И МАТЕМАТИЧЕСКАЯ

ФИЗИКА

Том 146, № 1

январь, 2006

(C) 2006 г.

И. К. Костов*, В. Б. Петкова ${ }^{\dagger}$

\title{
ОБЪЕМНЫЕ КОРРЕЛЯЦИОННЫЕ ФУНКЦИИ В ДВУМЕРНОЙ КВАНТОВОЙ ГРАВИТАЦИИ
}

\begin{abstract}
Объемные трех- и четырехточечные тахионные корреляторы в двумерной лиувиллевской гравитации с нерациональным центральным зарядом материи $c<1$ вычислены в рамках двух подходов, которые сравниваются между собой. Подход на основе непрерывной конформной теории поля использует действие на тахионах генераторов основного кольца (ground ring), деформированных с помощью экранирующих зарядов Лиувилля и материи. Как следствие, выведена общая формула для трехточечных структурных констант операторного разложения для материи. Дискретная формулировка теории является обобщением теорий $A D E$-струн, в которых таргет-пространство представляет собой полубесконечную цепочку точек.
\end{abstract}

Ключевые слова: некритические струны, конформная теория поля, двумерная гравитация, петлевой газ.

\section{1. ВВЕДЕНИЕ И ОБЗОР РЕЗУЛЬТАТОВ}

Давно известно, что операторные разложения физических операторов в эффективной двумерной конформной теории поля, описывающей квантовую лиувиллевскую гравитацию, сводятся по модулю БРСТ-коммутаторов к простым соотношениям "слияния" (fusion) [1]. Кроме того, имеются аргументы, показывающие, что операторы с нулевым духовым числом замыкаются в кольцо - "основное кольцо", которое, более того, сохраняет тахионные модули. Предполагается, что в рациональном случае оно совпадает с кольцом слияния минимальных теорий при $c<1$ (см., в частности, недавнюю работу [2]). Действие основного кольца на тахионных модулях использовалось для вывода функциональных рекуррентных тождеств для тахионных корреляционных функций [3]-[5]. В данной работе (более подробное изложение см. в [6]) мы возвращаемся к этому подходу и распространяем его на построение объемных тахионных корреляторов. Кроме того, мы рассматриваем нерациональную конформную теорию поля двумерной квантовой гравитации, в которой вырожденные

\footnotetext{
*Service de Physique Théorique, CNRS - URA 2306, C.E.A. - Saclay, F-91191 Gif-Sur-Yvette, France

${ }^{\dagger}$ Institute for Nuclear Research and Nuclear Energy, 72 Tsarigradsko Chaussée, 1784 Sofia, Bulgaria. E-mail: petkova@inrne.bas.bg
} 
поля ограничены на диагональ полубесконечной таблицы Каца. Микроскопическая реализация этой диагональной конформной теории поля является нерациональным расширением теорий $A D E$-струн, введенных в работе [7] для полубесконечного дискретного таргет-пространства. Представление микроскопической теории в форме петлевого газа приводит к диаграммной технике в таргет-пространстве [8], которая позволяет эффективно вычислять тахионные корреляционные функции [9], [10]. Мь установим согласие между конформной теорией поля и дискретными результатами.

Нерациональная двумерная гравитация: эффективное действие, локальные поля (тахионы), основное кольцо. Эффективное действие евклидовой лиувиллевской гравитации является возмущением гауссова действия

$$
\mathcal{A}^{\text {free }}=\frac{1}{4 \pi} \int d^{2} x\left[(\nabla \phi)^{2}+(\nabla \chi)^{2}+\left(Q \phi+i e_{0} \chi\right) \widehat{R}+2\left(\mathbf{b} \nabla_{\bar{z}} \mathbf{c}+\overline{\mathbf{b}} \nabla_{z} \overline{\mathbf{c}}\right)\right]
$$

для полей Лиувилля $\phi$, материи $\chi$ и пары репараметризационнных духов. Фоновые заряды $Q=1 / b+b$ и $e_{0}=1 / b-b$ параметризованы вещественной переменной $b$, так что полный центральный заряд этой конформной теории тривиален:

$$
c_{\text {tot }} \equiv c_{\mathrm{L}}+c_{\mathrm{m}}+c_{\text {ghostS }}=\left[13+6\left(b^{2}+\frac{1}{b^{2}}\right)\right]+\left[13-6\left(b^{2}+\frac{1}{b^{2}}\right)\right]-26=0 .
$$

Нормированное произведение вертексных операторов полей Лиувилля и материи полной размерности $(1,1)$

$$
\begin{gathered}
\frac{\gamma\left(1-\alpha^{2}+e^{2}\right)}{\pi} e^{2 i e \chi} e^{2 \alpha \phi}=\frac{1}{\pi} \gamma\left(\epsilon b^{\epsilon} P\right) e^{i\left(e_{0}-P\right) \chi+(Q-\epsilon P) \phi}=V_{\alpha}^{\epsilon}, \quad \epsilon= \pm 1, \\
e\left(e-e_{0}\right)+\alpha(Q-\alpha)=1 \Rightarrow e=\alpha-b \quad \text { или } \quad e=-\alpha+\frac{1}{b}
\end{gathered}
$$

(т.е. тахионы "на массовой оболочке") является примером физических БРСТинвариантных операторов [11]. Они получаются из (1.3) или посредством интегрирования по мировой поверхности (в качестве которой берется сфера), или с помощью умножения на духовое поле с $\overline{\mathbf{c}}$ размерности $(-1,-1)$ :

$$
\mathcal{T}_{P}^{( \pm)}=T_{\alpha}^{ \pm} \equiv \int V_{\alpha}^{ \pm} \quad \text { или } \quad \mathcal{W}_{P}^{( \pm)}=W_{\alpha}^{ \pm} \equiv \mathbf{c} \overline{\mathbf{c}} V_{\alpha}^{ \pm}
$$

Параметры $P$ и $\epsilon$ в (1.3) интерпретируются как импульс и киральность тахиона в таргет-пространстве. В нормировке "на хвостовые множители" в $(1.3)$ имеем $\gamma(x)=$ $\Gamma(x) / \Gamma(1-x)$.

"Основное кольцо" БРСТ-инвариантых операторов, имеющих нулевую размерность и нулевое духовое число [1], порождено двумя операторами

$$
\begin{aligned}
& a_{-}=\left|\mathbf{b c}-b^{-1} \partial_{z}(\phi+i \chi)\right|^{2} e^{-b(\phi-i \chi)}, \\
& a_{+}=\left|\mathbf{b c}-b \partial_{z}(\phi-i \chi)\right|^{2} e^{-b^{-1}(\phi+i \chi)} .
\end{aligned}
$$

В отличие от тахионов операторы (1.6) построены из вертексных операторов Лиувилля и материи, каждый из которых соответствует вырожденным представлениям алгебры Вирасоро. Рассмотрим деформации этих свободнополевых операторов, 
определяемые парой “экранирующиих зарядов” Лиувилля и материи, т.е. действием взаимодействия

$$
\begin{aligned}
& \mathcal{A}_{\text {int }}=\int\left(\mu_{\mathrm{L}} e^{2 b \phi}+\mu_{\mathrm{m}} e^{-2 i b \chi}\right)=\lambda_{\mathrm{L}} \mathcal{T}_{e_{0}}^{(+)}+\lambda_{\mathrm{m}} \mathcal{T}_{Q}^{(+)}, \\
& \tilde{\mathcal{A}}_{\text {int }}=\int\left(\tilde{\mu}_{\mathrm{L}} e^{2 \phi / b}+\tilde{\mu}_{\mathrm{m}} e^{2 i \chi / b}\right)=\tilde{\lambda}_{\mathrm{L}} \mathcal{T}_{e_{0}}^{(-)}+\tilde{\lambda}_{\mathrm{m}} \mathcal{T}_{-Q}^{(-)} .
\end{aligned}
$$

Константы взаимодействия, перенормированные хвостовыми множителями в (1.3), имеют вид

$$
\begin{array}{ll}
\lambda_{\mathrm{L}}=\pi \gamma\left(b^{2}\right) \mu, & \tilde{\lambda}_{\mathrm{L}}=\pi \gamma\left(\frac{1}{b^{2}}\right) \tilde{\mu}=\lambda_{\mathrm{L}}^{1 / b^{2}}, \\
\lambda_{\mathrm{m}}=\pi \gamma\left(-b^{2}\right) \mu_{\mathrm{m}}, & \tilde{\lambda}_{\mathrm{m}}=\pi \gamma\left(-\frac{1}{b^{2}}\right) \tilde{\mu}_{\mathrm{m}}=\left(\lambda_{\mathrm{m}}\right)^{-1 / b^{2}} .
\end{array}
$$

Четыре члена в (1.7), (1.8) являются частными примерами (интегралов от) тахионных вертексных операторов. Мы рассмотрим также другое возмущение действия (1.1), в котором экранирующиие заряды материи заменены парой тахионов, получаемых как отраженные относительно материи $(e, \alpha) \rightarrow\left(e_{0}-e, \alpha\right)$ образы двух лиувиллевских экранирующих зарядов:

$$
\mathcal{A}_{\text {int }}^{\text {diag }}=\lambda_{\mathrm{L}}\left(\mathcal{T}_{e_{0}}^{(+)}+\lambda_{\mathrm{m}} \tilde{\lambda}_{\mathrm{m}} \mathcal{T}_{-e_{0}}^{(-)}\right)+\tilde{\lambda}_{\mathrm{L}}\left(\mathcal{T}_{e_{0}}^{(-)}+\lambda_{\mathrm{m}} \tilde{\lambda}_{\mathrm{m}} \mathcal{T}_{-e_{0}}^{(+)}\right) .
$$

\section{2. ТАХИОНАЯ ТРЕХТОЧЕЧНАЯ ФУНКЦИЯ КАК ПРОИЗВЕДЕНИЕ ЛИУВИЛЛЕВСКОЙ И МАТЕРИАЛЬНОЙ ТРЕХТОЧЕЧНЫХ ФУНКЦИЙ}

В $n$-точечных тахионных корреляторах $n-3$ вертексных операторов интегрируются по мировой поверхности, а три располагаются, как обычно, в произвольных точках, например 0,1 и $\infty$. трехточечная функция факторизуется в произведение трехточечных констант операторного произведения для операторов материи и Лиувилля:

$$
G_{3}^{\varepsilon_{1} \varepsilon_{2} \varepsilon_{3}}\left(\alpha_{1}, \alpha_{2}, \alpha_{3}\right)=\left\langle\mathcal{W}_{P_{1}}^{\left(\varepsilon_{1}\right)} \mathcal{W}_{P_{2}}^{\left(\varepsilon_{2}\right)} \mathcal{W}_{P_{3}}^{\left(\varepsilon_{3}\right)}\right\rangle=\frac{C^{\mathrm{L}}\left(\alpha_{1}, \alpha_{2}, \alpha_{3}\right) C^{\mathrm{m}}\left(e_{1}, e_{2}, e_{3}\right)}{\pi^{3} \prod_{j=1}^{3} \gamma\left(\alpha_{j}^{2}-e_{j}^{2}\right)} .
$$

Здесь $\alpha_{i}$ и $e_{i}$ - решения условий массовой оболочки (1.4). Для лиувиллевской трехточечной константы мы примем выражение, найденное в работах [12]:

$$
C^{\mathrm{L}}\left(\alpha_{1}, \alpha_{2}, \alpha_{3}\right)=\left(\lambda_{\mathrm{L}}^{1 / b} b^{2 e_{0}}\right)^{Q-\alpha_{1}-\alpha_{2}-\alpha_{3}} \frac{\Upsilon_{b}(b) \Upsilon_{b}\left(2 \alpha_{1}\right) \Upsilon_{b}\left(2 \alpha_{2}\right) \Upsilon_{b}\left(2 \alpha_{3}\right)}{\Upsilon_{b}\left(\alpha_{123}-Q\right) \Upsilon_{b}\left(\alpha_{23}^{1}\right) \Upsilon_{b}\left(\alpha_{13}^{2}\right) \Upsilon_{b}\left(\alpha_{12}^{3}\right)},
$$

где используются обозначения $\alpha_{12}^{3}=\alpha_{1}+\alpha_{2}-\alpha_{3}, \quad \alpha_{123}=\alpha_{1}+\alpha_{2}+\alpha_{3}$ и т.д. Общая трехточечная константа операторного произведения в теории материи с $c<1$ имеет вид

$$
\begin{aligned}
C^{\mathrm{m}}\left(e_{1}, e_{2}, e_{3}\right) & =\left(\lambda_{\mathrm{m}}^{1 / b} b^{2 Q}\right)^{e_{1}+e_{2}+e_{3}-e_{0}} \frac{\widehat{\Upsilon}_{b}(0) \widehat{\Upsilon}_{b}\left(2 e_{1}\right) \widehat{\Upsilon}_{b}\left(2 e_{2}\right) \widehat{\Upsilon}_{b}\left(2 e_{3}\right)}{\widehat{\Upsilon}_{b}\left(e_{123}-e_{0}\right) \widehat{\Upsilon}_{b}\left(e_{23}^{1}\right) \widehat{\Upsilon}_{b}\left(e_{13}^{2}\right) \widehat{\Upsilon}_{b}\left(e_{12}^{3}\right)}= \\
& =\frac{\lambda_{\mathrm{L}}^{\left(Q-\sum_{i} \alpha_{i}\right) / b} \lambda_{\mathrm{m}}^{-\left(e_{0}-\sum_{i} e_{i}\right) / b}}{b^{\sum_{i} \epsilon_{i}} \prod_{i=1}^{3} \gamma\left(b^{\epsilon_{i}}\left(Q-2 \alpha_{i}\right)\right)} \frac{1}{C^{\mathrm{L}}\left(\alpha_{1}, \alpha_{2}, \alpha_{3}\right)}, \quad \alpha_{i}=\epsilon_{i} e_{i}+b^{\epsilon_{i}} .
\end{aligned}
$$


Вторая строчка имеет место при любом выборе трех знаков $\epsilon_{i}$ с использованием свойств отражения лиувиллевской константы (2.2). Функция в первой строчке определяется как

$$
\widehat{\Upsilon}_{b}(x)=\frac{1}{\Upsilon_{b}(x+b)}=\frac{1}{\Upsilon_{b}(-x+1 / b)}=\widehat{\Upsilon}_{b}\left(e_{0}-x\right)=\widehat{\Upsilon}_{1 / b}(-x)
$$

и удовлетворяет функциональным соотношениям

$$
\widehat{\Upsilon}_{b}(x-b)=\gamma(b x) b^{1-2 b x} \widehat{\Upsilon}_{b}(x), \quad \widehat{\Upsilon}_{b}\left(x+\frac{1}{b}\right)=\gamma\left(-\frac{1}{b} x\right) b^{-1-2 x / b} \widehat{\Upsilon}_{b}(x) .
$$

Ее логарифм допускает интегральное представление, подобное представлению для $\ln \Upsilon_{b}$, но с заменой $Q$ на $e_{0}$ (а потому инвариантное относительно замены $b \rightarrow-1 / b$ ), которое сходится (при $b>0)$ в полосе $-b<\operatorname{Re} x<1 / b$. Нормировка в выражении (2.3) фиксированна выбором

$$
C^{\mathrm{m}}\left(e_{1}, e_{2}, e_{3}\right)=1 \quad \text { при } \quad \sum_{i} e_{i}=e_{0} .
$$

При $\sum_{i} e_{i}-e_{0}=m b-n / b$, где $n, m-$ неотрицательные целые числа, выражение $(2.3)$ является конечным для $b^{2}$ и $e_{i}$ в общем положении и воспроизводит с точностью до степеней $\left(-\mu_{\mathrm{m}}\right)^{m}\left(-\tilde{\mu}_{\mathrm{m}}\right)^{n}$ трехточечную константу Доценко-Фатеева (см. формулу (В.10) работы [13]). Таким образом, оно воспроизводит пертурбативный порядок $(m, n)$ действия материи с учетом экранирующих зарядов материи. Вывод формулы (2.3) повторяет вывод для лиувиллевского случая, рассморенного в работе [14], где формула (2.2) определялась для положительных иррациональных $b^{2}$ как единственное (гладкое) решение пары функциональных соотношений (дальнейшие подробности см. в работе [6] $)^{1)}$.

Подставляя два выражения (2.2) и (2.3) в (2.1), получаем простое выражение для тахионной трехточечной функции:

$$
G_{3}^{\varepsilon_{1} \varepsilon_{2} \varepsilon_{3}}\left(\alpha_{1}, \alpha_{2}, \alpha_{3}\right)=\frac{1}{\pi^{3} b^{\varepsilon_{1}+\varepsilon_{2}+\varepsilon_{3}}} \lambda_{\mathrm{L}}^{\left(Q-\sum_{i} \alpha_{i}\right) / b} \lambda_{M}^{-\left(e_{0}-\sum_{i} e_{i}\right) / b},
$$

которое воспроизводит известный результат (см. работу [16] и приведенную там библиографию). За исключением степени $\lambda_{\mathrm{m}}$ это выражение не зависит от присутствия экранирующих зарядов материи. С другой стороны, константа (2.2) радикально упрощается до выражения (2.7) уже с использованием условия "нейтральности" для зарядов материи в уравнении (2.6), которое одновременно является условием для лиувиллевских зарядов.

Статистическая сумма $Z_{\mathrm{L}}\left(\lambda_{\mathrm{L}}, \lambda_{\mathrm{m}}, b\right)$ традиционно определяется тем, что ее третья производная по $\lambda_{\mathrm{L}}$ отождествляется с $-G_{3}^{+++}(b, b, b)$. трехточечные корреляторы, нормированные с помощью $Z_{\mathrm{L}}\left(\lambda_{\mathrm{L}}, \lambda_{\mathrm{m}}, b\right)$, удовлетворяют различным тождествам дуальности.

Формула (2.7), имеющая вид произведения теории поля материи на теорию поля Лиувилля, выполняется для импульсов в общем положении при допущениях о

\footnotetext{
1) Вывод константы для материи был независимо выполнен Ал. Замолодчиковым [15].
} 
нормировке для двух констант в произведении. Она, однако, является формальной, представляя собой выражение $0 \times \infty$ в сингулярных точках констант. По тем же причинам в общем случае не следует ожидать выполнения простого соотношения, включающего в себя функциональные соотношения для полей материи и Лиувилля. Ввиду этих наблюдений мы заново рассмотрим задачу об определении тахионной трехточечной функции. Дело сводится к паре уравнений, которые выводятся ниже как часть набора рекуррентнных тождеств для $n$-точечных тахионных корреляторов. Эти уравнения более слабые, чем комбинация функциональных тождеств для системы "материя плюс Лиувилль", и выражение (2.7) является всего лишь простейшим их решением. Для нормированного множителя в общем выражении

$$
\mathcal{G}^{\varepsilon_{1} \varepsilon_{2} \varepsilon_{3}}\left(P_{1}, P_{2}, P_{3}\right)=\frac{\lambda_{\mathrm{L}}^{\left(\sum_{i} \varepsilon_{i} P_{i}-Q\right) /(2 b)} \lambda_{\mathrm{m}}^{\left(e_{0}-\sum_{i} P_{i}\right) /(2 b)}}{\pi^{3} b^{\varepsilon_{1}+\varepsilon_{2}+\varepsilon_{3}}} N_{P_{1}, P_{2}, P_{3}}
$$

эти уравнения имеют вид

$$
N_{P_{1}+b^{\epsilon}, P_{2}, P_{3}}+N_{P_{1}-b^{\epsilon}, P_{2}, P_{3}}=N_{P_{1}, P_{2}+b^{\epsilon}, P_{3}}+N_{P_{1}, P_{2}-b^{\epsilon}, P_{3}}, \quad \epsilon= \pm 1 .
$$

В теории, соответствующей диагональному действию (1.10), трехточечная функция определяется уравнением того же типа, но со сдвигами импульсов на $e_{0}$.

\section{3. ДЕЙСТВИЕ ОСНОВНОГО КОЛЬЦА НА ТАХИОНАХ}

Наиболее существенное свойство операторов $a_{ \pm}$в (1.6) состоит в том, что их производные $\partial_{z} a_{ \pm}$и $\partial_{\bar{z}} a^{ \pm}$БРСТ-точны: $\partial_{z} a_{-}=\left\{Q_{\text {БРст }}, \mathbf{b}_{-1} a_{-}\right\}$. Поэтому любая амплитуда, в которую входит $a_{ \pm}$и другие БРСТ-инвариантые операторы, не зависит от положения операторов $a_{ \pm}$. Это свойство позволяет записать рекуррентные уравнения для тахионных корреляционных функций, используя тот факт, что БРСТинвариантные операторы $W_{\alpha}^{ \pm}$образуют модуль над основным кольцом с точностью до коммутаторов с БРСТ-зарядом [3]:

$$
\begin{gathered}
a_{-} W_{\alpha}^{-}=-W_{\alpha-b / 2}^{-}, \quad a_{+} W_{\alpha}^{+}=-W_{\alpha-1 /(2 b)}^{+} \\
a_{-} W_{\alpha}^{+}=a_{+} W_{\alpha}^{-}=0
\end{gathered}
$$

Оба соотношения следуют из операторных разложений для свободных полей и деформируются в присутствии интегралов от тахионных вертексных операторов. Таким образом, второе соотношение приводится к виду [4]

$$
a_{-} W_{\alpha}^{+} T_{\alpha_{1}}^{+}=W_{\alpha+\alpha_{1}-b / 2}^{+}, \quad a_{+} W_{\alpha}^{-} T_{\alpha_{1}}^{-}=W_{\alpha+\alpha_{1}-1 /(2 b)}^{-} .
$$

Специальный пример получается из (3.2), если выбрать $P_{1}=e_{0}$, т.е. $\alpha_{1}=b$ и $\alpha_{1}=1 / b$ в первом и втором соотношениях, соответственно. В этом случае $T_{\alpha_{1}}^{ \pm}$совпадают с лиувиллевскими членами взаимодействия в (1.7) и (1.8). Рассмотрение их в качестве возмущений сводится к модификации исходных генераторов кольца следующим образом [5]:

$$
a_{-} \rightarrow a_{-}\left(1-\lambda_{\mathrm{L}} T_{b}^{+}+\cdots\right), \quad a_{+} \rightarrow a_{+}\left(1-\tilde{\lambda}_{\mathrm{L}} T_{1 / b}^{-}+\cdots\right) .
$$


Другая деформация генераторов кольца (1.6), включающая экранирующие заряды материи, соответствует выбору $\alpha_{1}=0$, т.е. $P_{1}=Q$ или $P_{1}=-Q$. Более того, действие генераторов кольца нетривиально на некоторых специальных двойных интегралах:

$$
a_{-} W_{\alpha}^{-} T_{\alpha_{1}}^{+} T_{b-\alpha_{1}}^{+}=-W_{\alpha+b / 2}^{-}, \quad a_{+} W_{\alpha}^{+} T_{\alpha_{1}}^{-} T_{1 / b-\alpha_{1}}^{-}=-W_{\alpha+1 /(2 b)}^{+}
$$

Выбор $\alpha_{1}=b$ в первом и $\alpha_{1}=1 / b$ во втором из соотношений (3.4) соответствует комбинации возмущений первого порядка в теории материи и Лиувилля.

Итак, соотношения (3.1) деформируются следующим образом (мы сохраняем те же обозначения для полностью деформированных генераторов кольца):

$$
\begin{gathered}
a_{-} W_{\alpha}^{+}=-\lambda_{\mathrm{L}} W_{\alpha+b / 2}^{+}-\lambda_{\mathrm{m}} W_{\alpha-b / 2}^{+}, \\
a_{-} W_{\alpha}^{-}=-W_{\alpha-b / 2}^{-}-\lambda_{\mathrm{L}} \lambda_{\mathrm{m}} W_{\alpha+b / 2}^{-}, \\
a_{+} W_{\alpha}^{-}=-\tilde{\lambda}_{\mathrm{L}} W_{\alpha+1 /(2 b)}^{-}-\tilde{\lambda}_{\mathrm{m}} W_{\alpha-1 /(2 b)}^{-}, \\
a_{+} W_{\alpha}^{+}=-W_{\alpha-1 /(2 b)}^{+}-\tilde{\lambda}_{\mathrm{L}} \tilde{\lambda}_{\mathrm{m}} W_{\alpha+1 /(2 b)}^{+} .
\end{gathered}
$$

Для оператора $a_{+} a_{-}$, возмущенного диагональным членом взаимодействия в действии (1.10) (обозначаемым как $A_{+-}$), получаем похожее соотношение со сдвигом на $e_{0} / 2$, которое мы запишем в терминах импульсов $P=\epsilon(Q-2 \alpha)=e_{0}-2 e$ :

$$
\begin{aligned}
& A_{+-} \mathcal{W}_{P}^{(+)}=\lambda_{\mathrm{L}} \mathcal{W}_{P+e_{0}}^{(+)}+\tilde{\lambda}_{\mathrm{L}} \lambda_{\mathrm{m}}^{-e_{0} / b} \mathcal{W}_{P-e_{0}}^{(+)}, \\
& A_{+-} \mathcal{W}_{\alpha}^{(-)}=\tilde{\lambda}_{\mathrm{L}} W_{P+e_{0}}^{(-)}+\lambda_{\mathrm{L}} \lambda_{\mathrm{m}}^{-e_{0} / b} \mathcal{W}_{P-e_{0}}^{(-)}
\end{aligned}
$$

Тождества (3.5), (3.6) обобщают частные результаты работ [3]-[5] для $\lambda_{\mathrm{m}}=0=\tilde{\lambda}_{\mathrm{m}}$. Только два члена в каждом из этих соотношений (из четырех членов в операторном разложении фундаментальных вертексных операторов материи и Лиувилля в (1.6)) сохраняют условия (1.4). Имеются дальнейшие обобщения (3.4) на случай $2 m$ интегралов от тахионных операторов одной и той же киральности, если сумма лиувиллевских экспонент равна $m b$ и $m / b$, соответственно. Если все импульсы соответствуют экранирующим зарядам или материи, или Лиувилля, то соответствующие коэффициенты операторного разложения обращаются в нуль при $m>1$, откуда следует, что новых поправок к двучленному действию (3.5), (3.6) генераторов кольца нет. С другой стороны, диагональное действие (1.10) задумано таким образом, чтобы проектировать четрырехчленное действие $a_{-} a_{+}$-деформации на два члена из (3.7).

\section{4. РЕШЕНИЯ ФУНКЦИОНАЛЬНОГО УРАВНЕНИЯ ДЛЯ ТРЕХТОЧЕЧНОЙ ФУНКЦИИ}

Используя соотношения (3.5), (3.6) в четырехточечной функции с тремя тахионами, получаем функциональные уравнения (2.9) для тахионных трехточечных функций. Аналогичное соотношение со сдвигами на $e_{0}$ следует из (3.7). Мы приведем здесь некоторые другие, помимо (2.7), примеры решения этих уравнений. 
Ограничиваясь тахионами, занумерованными вырожденными представлениями материи,

$$
e_{0}-2 e=P= \pm\left(-m b+\frac{n}{b}\right), \quad m, n \in \mathbb{Z}_{>0}
$$

и, в частности, постулируя, что тахионы фиксированной киральности, занумерованные граничными линиями при $m=0$ или $n=0$, обращаются в нуль, получаем в качестве решений уравнения (2.9) произведение кратностей в разложениях тензорных произведений неприводимых $s l(2)$-представлений размерностей $m_{i}, n_{i}{ }^{2}$ :

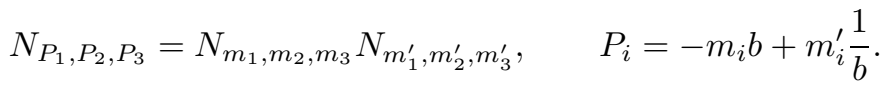

Здесь

$$
\begin{gathered}
N_{m_{1}, m_{2}, m_{3}}=\left\{\begin{array}{ccc}
1, & \text { если } & \left|m_{1}-m_{2}\right|+1 \leqslant m_{3} \leqslant m_{1}+m_{2}-1 \\
0 & \text { в других случаях }
\end{array}\right. \\
N_{m_{1}+1, m_{2}, m_{3}}-N_{m_{1}-1, m_{2}, m_{3}}=N_{m_{1}+m_{2}, m_{3}, 1}-N_{m_{1}-m_{2}, m_{3}, 1} .
\end{gathered}
$$

Правая часть разностного тождества в (4.3) указывает на отклонение от простого функционального соотношения вида произведения теорий материии Лиувилля. Кратность (4.2) можно альтернативным образом отождествить с решением уравнения (2.9) для импульсов, занумерованных вырожденными представлениями Лиувилля. В диагональном случае $m=n$ решением следующего из (3.7) уравнения является один из таких множителей, если отождествить $P_{i}=\epsilon_{i} m_{i} e_{0}$. Другим решением (на этот раз с $P \in \mathbb{R}$ ) является выражение, двойственное к (4.2):

$$
\begin{gathered}
N_{P_{1}, P_{2}, P_{3}}=\sum_{m=0} \sum_{n=0}\left(2 \sin \left(\pi m e_{0} b\right) \sin \left(\pi n \frac{e_{0}}{b}\right)\right)^{2} \chi_{P_{1}}(m, n) \chi_{P_{2}}(m, n) \chi_{P_{3}}(m, n), \\
\chi_{P}(m, n)=\frac{\sin (\pi m P b)}{\sin \left(\pi m e_{0} b\right)} \frac{\sin (\pi n P / b)}{\sin \left(\pi n e_{0} / b\right)}=\chi_{-P}(m, n),
\end{gathered}
$$

в котором вырожденные представления материи (или Лиувилля) нумеруют дуальные (граничные) переменные. Другой пример при вещественном $P$ дается кратностью проектирования на диагональные заряды материи:

$$
\begin{gathered}
N_{P_{1}, P_{2}, P_{3}}=\sum_{l=0}^{\infty} \delta\left(P_{1}+P_{2}+P_{3}-(2 l+1) e_{0}\right) \\
N_{P_{1}+e_{0}, P_{2}, P_{3}}-N_{P_{1}-e_{0}, P_{2}, P_{3}}=\delta\left(P_{1}+P_{2}+P_{3}\right) .
\end{gathered}
$$

\footnotetext{
${ }^{2)} \mathrm{B}$ недавно проведенном заново [2] выводе этого результата для рационального $b^{2}$ вместо вычисления коэффициентов операторного разложения само основное кольцо отождествляется с кольцом слияния минимальных моделей с $c<1$.
} 


\section{5. ФУНКЦИОНАЛЬНЫЕ УРАВНЕНИЯ ДЛЯ ЧЕТЫРЕХТОЧЕЧНЫХ ТАХИОННЫХ АМПЛИТУД}

Интегралы от тахионных вертексных операторов в $n$-точечных функциях при $n \geqslant 4$ играют роль экранирующих зарядов, а из формул операторных разложений, таких как (3.2) и (3.4), следуют новые, помимо (3.5), (3.6), каналы для действия генераторов кольца. Это приводит к появлению дополнительных членов с менее чем $n$ полями; альтернативным образом ожидается, что эти "контактные" члены учитывают пропущенные БРСТ-коммутаторы в операторных тождествах (3.5), (3.6). Выпишем в качестве иллюстрации соотношения для четырехточечной функции $G_{4}^{-+++}\left(\alpha_{1}, \alpha_{2}, \alpha_{3}, \alpha_{4}\right)=\left\langle W_{\alpha_{1}}^{-} W_{\alpha_{2}}^{+} T_{\alpha_{3}}^{+} W_{\alpha_{4}}^{+}\right\rangle:$

$$
\begin{aligned}
G_{4}^{-+++} & \left(\alpha_{1}-\frac{b}{2}, \alpha_{2}, \alpha_{3}, \alpha_{4}\right)+\lambda_{\mathrm{L}} \lambda_{\mathrm{m}} G_{4}^{-+++}\left(\alpha_{1}+\frac{b}{2}, \alpha_{2}, \alpha_{3}, \alpha_{4}\right)- \\
& \quad-\lambda_{\mathrm{L}} G_{4}^{-+++}\left(\alpha_{1}, \alpha_{2}+\frac{b}{2}, \alpha_{3}, \alpha_{4}\right)-\lambda_{\mathrm{m}} G_{4}^{-+++}\left(\alpha_{1}, \alpha_{2}-\frac{b}{2}, \alpha_{3}, \alpha_{4}\right)= \\
=- & G_{3}^{-++}\left(\alpha_{1}, \alpha_{2}+\alpha_{3}-\frac{b}{2}, \alpha_{4}\right)+ \\
& +\left(\lambda_{\mathrm{L}} \delta_{\alpha_{3}, 0}+\lambda_{\mathrm{m}} \delta_{\alpha_{3}, b}\right) G_{3}^{-++}\left(\alpha_{1}+\frac{b}{2}, \alpha_{2}, \alpha_{4}\right) .
\end{aligned}
$$

Выбор того, какой из трех операторов с положительной киральностью представлен интегралом от вертекса, не должен быть существенным - это приводит к набору соотношений, получаемых из (5.1) перестановками величин $\alpha_{s}, s=2,3,4$. Тождество, аналогичное (5.1), для функции $G_{4}^{+---}$с противоположными киральностями, следующее из (3.6), получается заменами $b \rightarrow 1 / b, \lambda_{\mathrm{L}} \rightarrow \tilde{\lambda}_{\mathrm{L}}, \lambda_{\mathrm{m}} \rightarrow \tilde{\lambda}_{\mathrm{m}}$. Аналогичные соотношения, но со сдвигами $e_{0} / 2$, получаются из диагонального действия кольца (3.7).

Ожидается, что соотношения для четырехточечных функций выполнены для тахионных импульсов в общем положении ${ }^{3)}$. Кроме двух специальных контактных членов в последней строке в (5.1), которые происходят из двойного интегрального соотношения (3.4), потенциально имеются и другие члены, соответствующие многократным интегралам, обобщающим (3.4) и таким, что не все $T_{\alpha_{i}}$ представлены экранирующими зарядами.

В отсутствие экранирующих зарядов материи, $e-e_{0}=0$, решения частично деформированного кольцевого соотношения, получаемого при $\lambda_{\mathrm{m}}=0$ в (3.5), воспроизводят четырехточечные функции, найденные другими средствами в работе [16]. В общем случае мы рассмотрим сначала трехточечные функции, дающиеся простым выражением (2.7). Мы ограничим один из импульсов $P_{2}=1 / b-(m+1) b$ на область вырожденных значений, налагая условие равенства нулю тахиона в граничной точке $P=1 / b$ из (4.1). Остающиеся три импульса предполагаются в общем положении. Тогда уравнение (5.1) решается рекуррентно, если учесть, что

\footnotetext{
3) Правая часть выражения (5.1) требует модификации, если также включить в рассмотрение возмущение дуальным действием (1.8), см. [6].
} 
функция $G_{4}^{-+++}\left(\alpha_{1}, b, \alpha_{3}, \alpha_{4}\right)$ задается производной по $\lambda_{\mathrm{L}}$ от трехточечной функции $G_{3}^{-++}\left(\alpha_{1}, \alpha_{3}, \alpha_{4}\right)$. Полагая $\alpha=\sum_{i} \alpha_{i}$ и $e=\sum_{i} e_{i}$, получим

$$
G_{4}^{-+++}\left(\alpha_{1}, \alpha_{2}=b+\frac{m b}{2}, \alpha_{3}, \alpha_{4}\right)=\frac{\lambda_{\mathrm{L}}^{(Q-\alpha) / b} \lambda_{m}^{-\left(e_{0}-e\right) / b}}{\pi^{3} b^{2}}(m+1)\left(\sum_{s \neq 2} \alpha_{s}-Q+\frac{m b}{2}\right),
$$

и аналогичное выражение для функции с противоположными киральностями.

Наш второй пример относится к случаю диагонального $m=n$ вырожденного представления материи с трехточечной функцией, определяемой кратностью (4.3). Решение для четырехточечной функции имеет структуру разложения на три канала, что дает обобщение формулы из работы [16] для случая тривиальной материи. Оно имеет вид (с опущенным универсальным общим множителем $\lambda_{\mathrm{L}}, \lambda_{\mathrm{m}}$ )

$$
\begin{gathered}
G_{4}\left(\alpha_{1}, \alpha_{2}, \alpha_{3}, \alpha_{4}\right)= \\
=-\frac{1}{2 \pi^{3} e_{0}}\left(\sum_{m=0}\left(N_{m_{1}, m_{2}, m}\left(\frac{Q}{3}-m e_{0}\right) N_{m, m_{3}, m_{4}}+\text { перестановки }\right)\right)= \\
=\frac{1}{\pi^{3} e_{0}} N_{m_{1}, m_{2}, m_{3}, m_{4}}\left(Q+b-\sum_{i} \alpha_{i}-\frac{1}{2} e_{0}\left(N_{m_{1}, m_{2}, m_{3}, m_{4}}-1\right)\right), \\
\alpha_{i}=\frac{Q}{2}-m_{i} \frac{e_{0}}{2} .
\end{gathered}
$$

Полагая, что наибольшим значением $m_{i}$ является, скажем, $m_{1}$, т.е. $m_{1} \geqslant m_{s}$, $s=2,3,4$, отождествим симметричную по четырем аргументам функцию (5.3) с коррелятором типа $G_{4}^{-+++}$. Контактный член дается линейной комбинацией трехточечных функций

$$
[N]_{m_{1}, m_{2}+m_{3}, m_{4}}=N_{m_{1}, m_{2}+m_{3}, m_{4}}-N_{m_{1},\left|m_{2}-m_{3}\right|, m_{4}},
$$

которая принимает значения $0, \pm 1$. Второй член в (5.4) отражает тот факт, что поля обращаются в нуль на границе $m=0$ диагонали (4.1) и могут быть интерпретированы как антисимметричные по $P$ комбинации, так что тахион и образ его материального (плюс лиувиллевского) отражения отождествляются.

\section{6. МИКРОСКОПИЧЕСКАЯ РЕАЛИЗАЦИЯ ДИАГОНАЛЬНОЙ КОНФОРМНОЙ ТЕОРИИ ПОЛЯ}

6.1. SOS-модель как дискретизация гауссова поля материи. Если поле материи является свободным гауссовым полем с фоновым зарядом, т.е. если $\lambda_{\mathrm{m}}=0$, то двумерную гравитацию можно реализовать микроскопически как специальную SOS-модель (solid-on-solid) с комплексными больцмановскими весами [17]. Локальная флуктуирующая переменная в SOS-модели есть целочисленная высота $x \in \mathbb{Z}$, и конфигурации с допустимыми высотами таковы, что высоты в двух точках, являющихся ближайшими соседями, или совпадают, или различаются на \pm 1 . Поэтому каждая SOS-конфигурация определяет набор областей равной высоты, накрывающих двумерную решетку. Границы областей образуют узор непересекающихся петель на решетке. Таким образом, SOS-модель описывается также петлевым газом, 
т.е. ансамблем несамопересекающихся и взаимно непересекающихся петель, которые возникают на границах областей равной высоты.

Петлевой газ описывает целый класс решаемых моделей с функцией высоты, такие как RSOS-модели (restricted SOS) [18] и их $A D E$-обобщения [19], в которых таргет-пространство $\mathbb{T}$, где принимает значения переменная высоты, является ансамблем узлов графа Дынкина с простыми связями ( $A D E$-типа). Локальные больцмановские веса моделей с функцией высоты зависят от "массы" петель $M$ и от компонент $S=\left\{S_{x}\right\}_{x \in \mathbb{T}}$ собственного вектора матрицы инцидентности $A=\left\{A_{x, x^{\prime}}\right\}$ графа $\mathbb{T}$ :

$$
\sum_{x^{\prime}} A_{x, x^{\prime}} S_{x^{\prime}}=2 \cos \left(\pi p_{0}\right) S_{x}
$$

Для унитарных $A D E$-моделей это вектор Перрона-Фробениуса (ПФ).

Вес каждой конфигурации высот факторизуется в произведение весов связных областей, а петли представляются границами областей. Вес области $\mathcal{D}$ равен

$$
\Omega_{\mathcal{D}}(x)=\left(S_{x}\right)^{2-n}, \quad n=\# \text { границ области } \mathcal{D} \text {. }
$$

Кроме того, петли, или границы областей, берутся с весом, определяемым множителем $e^{-M \times д л и н а ~}$, аналогично каплям в модели Изинга. Сумму по высотам несложно вычислить, используя соотношение (6.1), и в результате каждая петля приобретает множитель $2 \cos \left(\pi p_{0}\right)$.

B SOS-модели роль вектора ПФ $S$ играет вектор

$$
S_{x}=\frac{1}{\sqrt{2}} e^{i \pi p_{0} x}, \quad x \in \mathbb{Z},
$$

где $p_{0}$ - любое вещественное число из интервала $[0,1]$. На основе некоторых аргументов, предложенных в статье [20], в работе [8] было предположено, что критическое поведение SOS-модели на решетке с дефектами кривизны описывается в непрерывном пределе теорией гауссова поля $\chi$ с действием (1.1). Переменная высоты $x$ и фоновый импульс $p_{0}$ связаны с гауссовым полем $\chi$ и фоновым электрическим зарядом $e_{0}=1 / b-b[21]^{4)}$ :

$$
p_{0}=\frac{1}{b^{2}}-1, \quad \chi=\frac{\pi x}{b} .
$$

Если SOS-модель рассматривается в ансамбле планарных графов с заданной топологией, то непрерывный предел описывается полным действием (1.1). Лиувиллевское взаимодействие в (1.7) управляется дополнительным множителем $e^{-\lambda_{\mathrm{L}} \times \text { площадь }}$ в больцмановских весах (6.2). В разреженной фазе $\lambda_{\mathrm{L}} \sim M^{2}$. Подробное описание SOS-модели, взаимодействующей с гравитацией, можно найти в работе [8].

6.2. Теория при $\lambda_{\mathrm{m}} \neq 0$ как SRSOS-модель (semi-restricted SOS). Можно привести аргументы в пользу того, что микроскопическая реализация теории (1.1), деформированной членом (1.10), дается для $b$ в общем положении “полуограничен-

\footnotetext{
4) Здесь мы рассматриваем только разреженную фазу петлевого газа. В плотной фазе соответствие имеет вид $p_{0}=1-b^{2}, \chi=\pi b x$.
} 
ной” моделью с функцией высоты, взаимодействующей с гравитацией, с таргетпространством $\mathbb{T}=\mathbb{Z}_{>0}$. Больцмановские веса определяются формулой (6.2) при

$$
S_{x}=\sqrt{2} \sin \left(\pi p_{0} x\right), \quad x \in \mathbb{Z}_{>0}
$$

Фоновый заряд и нормировка поля снова даются формулой (6.4) для всех значений $b$. Ниже мы увидим, что при этом отождествлении четырехточечные функции в “диагональной” конформной теории поля (1.10) и в петлевой SRSOS-модели совпадают с точностью до числового множителя.

Локальные операторы порядка в SOS- и SRSOS-моделях строятся путем подстановки волновых функций

$$
\psi_{p}(x)=e^{i \pi\left(p-p_{0}\right) x}, \quad \psi_{p}(x)=\frac{\sin (\pi p x)}{\sin \left(\pi p_{0} x\right)}
$$

соответственно.

В непрерывном пределе операторы (6.6) описываются конформными полями с размерностью

$$
\Delta_{p}=\frac{p^{2}-p_{0}^{2}}{4\left(1+p_{0}\right)}=\frac{\left(P^{2}-e_{0}^{2}\right)}{4}
$$

6.3. Диаграммная техника в таргет-пространстве. Представление петлевого газа позволяет построить диаграммную технику в таргет-пространстве для струнного интеграла по траекториям, описанную в работах [8], [10], [22]. $n$-Точечные функции даются суммой всех возможных фейнмановских диаграмм, составленных из вершин, пропагаторов, “головастиков" и хвостовых множителей. Правила состоят в том, что вершины можно присоединять или к головастикам, или к пропагаторам, или к хвостовым множителям. Запрещено соединять непосредственно две вершины или пропагатор с головастиком. Правила Фейнмана представлены на рис. 1

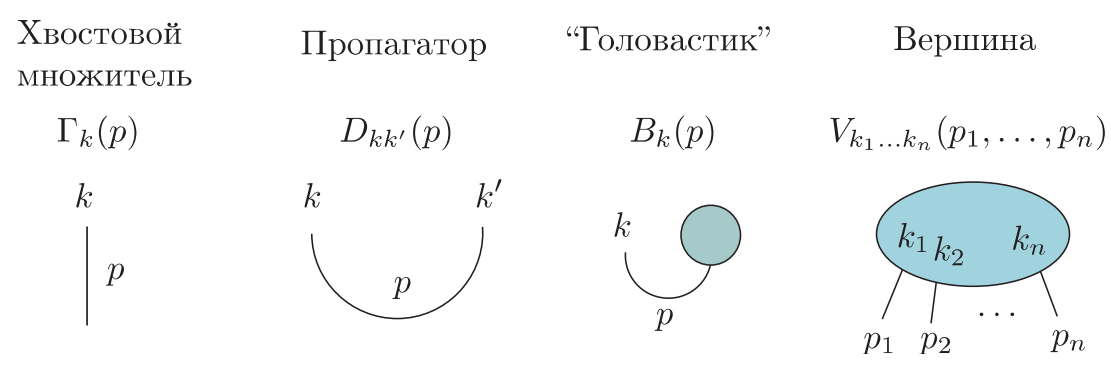

Рис. 1. Правила Фейнмана для корреляционных функций.

(здесь крайние точки линий изображены таким образом, что правила их склейки очевидны). Все элементы диаграммной техники зависят от двух типов квантовых чисел: периодического импульса $p+2 \equiv p$ в таргет-пространстве и неотрицательного целого $k$. 
- Пропагатор $D_{k, k^{\prime}}(p)$ :

$$
\begin{aligned}
& D_{00}(p)=-\left(|p|-\frac{1}{2}\right)\left(|p|-\frac{3}{2}\right), \\
& D_{01}(p)=-\frac{1}{2}\left(p^{2}-\frac{1}{4}\right)\left(|p|-\frac{3}{2}\right)\left(|p|-\frac{5}{2}\right)=D_{10}(p), \\
& D_{11}(p)=-\frac{1}{2}\left(p^{2}-\frac{1}{4}\right)\left(|p|-\frac{3}{2}\right)\left(|p|-\frac{5}{2}\right)\left[1+\frac{1}{3}\left(|p|-\frac{5}{2}\right)\left(|p|-\frac{7}{2}\right)\right], \ldots .
\end{aligned}
$$

- Вершины $V_{k_{1}, \ldots, k_{n}}\left(p_{1}, \ldots, p_{n}\right)$ :

$$
\begin{aligned}
V_{k_{1}, \ldots, k_{n}}\left(p_{1}, \ldots, p_{n}\right) & =\frac{\left(k_{1}+\cdots+k_{n}\right) !}{k_{1} ! \ldots k_{n} !} N_{p_{1}, \ldots, p_{n}}, \\
\text { где } \quad N_{p_{1}, \ldots, p_{n}} & =\sum_{x \in \mathbb{N}} S_{x}^{2} \prod_{j=1}^{n} \psi_{p_{j}}(x) .
\end{aligned}
$$

- Множители для внешних линий $\mathrm{S} \Gamma_{k}(p)$ :

$$
\Gamma_{k}(p)=\frac{1}{k !}\left(\frac{1}{2}+p\right)_{k}\left(\frac{1}{2}-p\right)_{k} .
$$

- Головастик $B_{k}(p)$ :

$$
B_{0}(p)=B_{1}(p)=0, \quad B_{k}(p)=-\delta\left(p, p_{0}\right) \Gamma_{k-1}(g), \quad k=2,3, \ldots,
$$

где $(a)_{n}=\Gamma(a+n) / \Gamma(a)-$ символ Похгаммера. Пропагатор, вершины и головастик определены выше для интервала $-1<p<1$. Их определение продолжается на произвольные значения $p$ требованием периодичности $p \rightarrow p+2$. Хвостовые множители не обязаны быть периодичными.

6.4. Общая формула для четырехточечной функции. Четырехточечная функция дается суммой трех фейнмановских диаграмм, показанных на рис. 2.

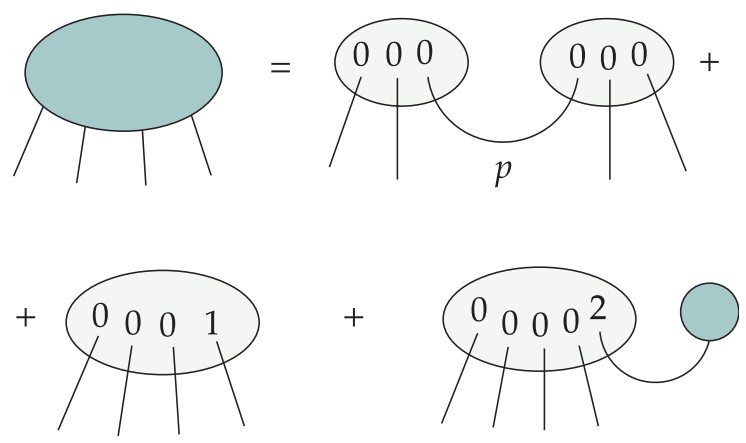

Рис. 2. Диаграммы для четырехточечной функции.

Каждая фейнмановская диаграмма представляет сумму членов, которые различаются перестановками внешних хвостов. 
Соответствующее аналитическое выражение имеет вид

$$
\begin{aligned}
& G\left(p_{1}, p_{2}, p_{3}, p_{4}\right)= \\
& =\int_{-1}^{1} d p\left[\Gamma_{0}\left(p_{1}\right) \Gamma_{0}\left(p_{2}\right) N_{p_{1} p_{2} p} D_{00}(p) N_{-p p_{3} p_{4}} \Gamma_{0}\left(p_{3}\right) \Gamma_{0}\left(p_{4}\right)+\text { перестановки }\right]+ \\
& \quad+\left[\Gamma_{0}\left(p_{1}\right) \Gamma_{0}\left(p_{2}\right) \Gamma_{0}\left(p_{3}\right) \Gamma_{1}\left(p_{4}\right)+\text { перестановки }\right] N_{p_{1} p_{2} p_{3} p_{4}}+ \\
& \quad+B_{2}\left(p_{0}\right) \Gamma_{0}\left(p_{1}\right) \Gamma_{0}\left(p_{2}\right) \Gamma_{0}\left(p_{3}\right) \Gamma_{0}\left(p_{4}\right) N_{p_{0} p_{1} p_{2} p_{3} p_{4}}
\end{aligned}
$$

Используя теперь соотношения (6.9)-(6.7), перепишем (6.11) в виде

$$
\begin{aligned}
& G\left(p_{1}, p_{2}, p_{3}, p_{4}\right)=\left[\left(1 \pm p_{0}\right)^{2}-\frac{1}{4}+\sum_{s=1}^{4}\left(\frac{1}{4}-p_{s}^{2}\right)\right] N_{p_{1}, p_{2}, p_{3}, p_{4}}+ \\
& \quad+\int_{-1}^{1} d p\left(N_{p_{1}, p_{2}, p} N_{-p, p_{3}, p_{4}}+N_{p_{1}, p_{3}, p} N_{-p, p_{2}, p_{4}}+N_{p_{1}, p_{4}, p} N_{-p, p_{2}, p_{3}}\right) \times \\
& \quad \times\left(|p|-\frac{1}{2}\right)\left(|p|-\frac{3}{2}\right) .
\end{aligned}
$$

Эта формула сраведлива для обеих моделей с функциями высоты, SOS и SRSOS. Она верна также для рациональных теорий $A D E$-струн, если интеграл по $p$ заменен на соответствующую дискретную сумму. В случае вырожденных полей рассмотрим операторы порядка (6.6) при $p=m p_{0}, \quad m=1,2,3, \ldots$ Интеграл в (6.12) заменяется на суммирование по положительным целым числам. В трехточечной кратности в (6.7) суммирование заменяется на интеграл, $x p_{0} \in[0,2]$, т.е. кратность имеет вид стандартного интегрального представления для (4.3). Возвращаясь к нормировке и обозначениям, используемым в теории на мировой поверхности, $P_{i}=\varepsilon_{i} b p_{i}=\varepsilon_{i} m_{i} b p_{0}, \quad e_{0}=b p_{0}$, мы в точности воспроизводим четырехточечную формулу (5.3).

Благодарности. Авторы благодарны Ал. Замолодчикову за полезные обсуждения, А. Белавину, Вл. Доценко, Ф. Шомерусу и Ж.-Б. Зюберу за интерес к данной работе и полезные замечания. Исследование поддержано со стороны European TMR Network EUCLID, контракт HPRN-CT-2002-00325, и Bulgarian National Council for Scientific Research, грант F-1205/02. И. К. Костов благодарен Institute for Advanced Study, Princeton, и Rutgers University за теплое гостеприимство во время выполнения части данной работы. В. Б. Петкова отмечает гостеприимство Service de Physique Théorique, CEA-Saclay.

\section{Список литературы}

[1] E. Witten. Nucl. Phys. B. 1992. V. 373. P. 187; hep-th/9108004.

[2] N. Seiberg, D. Shih. JHEP. 2004. V. 0402. P. 021.

[3] D. Kutasov, E. Martinec, N. Seiberg. Phys. Lett. B. 1992. V. 276. P. 437; hep-th/9111048.

[4] M. Bershadsky, D. Kutasov. Nucl. Phys. B. 1992. V. 382. P. 213; hep-th/9204049; S. Kachru. Mod. Phys. Lett. A. 1992. V. 7. P. 1419; hep-th/9201072.

[5] I. K. Kostov. Nucl. Phys. B. 2004. V. 689. P. 3; hep-th/0312301. 
[6] I. K. Kostov, V.B. Petkova. Non-rational 2D quantum gravity: ground ring versus loop gas. (in prep.).

[7] I. K. Kostov. Nucl. Phys. B. 1989. V. 326. P. 583.

[8] I. K. Kostov. Nucl. Phys. B. 1992. V. 376. P. 539; hep-th/9112059.

[9] I. K. Kostov, M. Staudacher. Phys. Lett. B. 1993. V. 305. P. 43; hep-th $/ 9208042$.

[10] S. Higuchi, I.K. Kostov. Phys. Lett. B. 1995. V. 357. P. 62; hep-th/9506022.

[11] F. David. Mod. Phys. Lett. A. 1988. V. 3. P. 1651; J. Distler, H. Kawai. Nucl. Phys. B. 1989. V. 321. P. 509.

[12] H. Dorn, H. J. Otto. Nucl. Phys. B. 1994. V. 429. P. 375; hep-th/9403141; A. B. Zamolodchikov, Al. B. Zamolodchikov. Nucl. Phys. B. 1996. V. 477. P. 577; hep-th $/ 9506136$.

[13] Vl.S. Dotsenko, V.A. Fateev. Nucl. Phys. B. 1985. V. 251. P. 691.

[14] J. Teschner. Phys. Lett. B. 1995. V. 363. P. 65; hep-th/9507109.

[15] Ал. Б. Замолодчиков. ТМФ. 2005. Т. 142. № 2. С. 218.

[16] P. Di Francesco, D. Kutasov. Nucl. Phys. B. 1992. V. 375. P. 119; hep-th/9109005

[17] B. Nienhuis. Coulomb gas formulation of 2-d phase transitions. In: Phase Transitions and Critical Phenomena. V. 11. Eds. C. C. Domb, J. L. Lebowitz. New York: Acad. Press, 1987. P. 1.

[18] G. E. Andrews, R. J. Baxter, P. J. Forrester. J. Stat. Phys. 1984. V. 35. P. 193.

[19] V. Pasquier. Nucl. Phys. B. 1987. V. 285. P. 162.

[20] O. Foda, B. Nienhuis. Nucl. Phys. B. 1989. V. 324. P. 643.

[21] I. K. Kostov, B. Ponsot, D. Serban. Nucl. Phys. B. 2004. V. 683. P. 309; hep-th/0307189.

[22] V. A. Kazakov, I. K. Kostov. Nucl. Phys. B. 1992. V. 386. P. 520; hep-th $/ 9205059$. 